\title{
Review
}

\section{Non-Destructive Techniques for Quality Evaluation of Intact Fruits and Vegetables}

\author{
Shyam Narayan JHA and Takahisa MATSUOKA \\ Faculty of Agriculture, Kochi University, B200 Monobe, Nankoku, 783 Japan
}

Received August 8, 2000; Accepted September 19, 2000

\begin{abstract}
Consumers are now more conscious about quality and source of their foods. Attempts made to determine the quality of food materials are numerous, but most of them are destructive in nature. In recent years, nondestructive methods of quality evaluation have gained momentum and a considerable attempts have been made to develop them. This paper reviews application of these non-destructive methods: magnetic resonance imaging, x-ray computer tomography, near infrared spectroscopy and a few other important techniques for determination of different quality attributes with emphasis on fruits and vegetables, and discusses their pros and cons for practical exploitation.
\end{abstract}

Keywords: non-destructive methods, quality evaluation, MRI, x-ray CT, NIR spectroscopy, fruits and vegetables.

Fruits and vegetables are increasing in popularity in the daily diets of people of both developed and developing countries. Product quality and quality evaluation methods are naturally extremely important. The decisions concerning harvesting, maturity, ripeness and quality are based mostly on subjective and visual inspection of the fruit's external appearance. Several nondestructive techniques for quality evaluation have been developed based on the detection of various physical properties that correlate well with certain factors of a product. The quality of fruits and vegetables is mostly based on size, shape, color, gloss, flavor, firmness, texture, taste and freedom from external as well as internal defects. Numerous techniques for evaluating the above external quality factors are now available commercially. Internal quality factors such as maturity, sugar content, acidity, oil content, and internal defects, however, are difficult to evaluate. Methods are needed to better predict the internal quality of fruits and vegetables without destroying them. Recently, there has been an increasing interest in non-destructive methods of quality evaluation, and a considerable amount of effort has been made in that direction. But the real problem is how these methods are to be exploited practically and what the difficulties are in implementing them. The objective of the present paper is thus to review the application of the most recent non-destructive methods such as nuclear magnetic resonance, $\mathrm{x}$-ray computed tomography, near-infrared spectroscopy and some other important methods and to evaluate their pros and cons for suitability in commercial application.

\section{Nuclear magnetic resonance (NMR) techniques}

The nuclear magnetic resonance technique, often referred as magnetic resonance imaging (MRI), involves resonant magnetic energy absorption by nuclei placed in an alternating magnetic field. The amount of energy absorbed by the nuclei is directly proportional to the number of a particular nucleus in the sample such as the protons in water or oil. The theory of NMR is presented in detail elsewhere (Farrar \& Becker, 1971). The basic

E-mail: snjha@cc.kochi-u.ac.jp concepts, types of pulsed experiments and the type of information that can be extracted from these experiments are described. Information on experimentation, assembling hardware, conducting laboratory tests and interpreting the results is also available from Fukushima and Roeder (1981). These authors also provided detailed theory for better understanding of what a scientist should seek and what he might expect to find out by using NMR.

There are many applications of NMR in agriculture (Rollwitz, 1984). The simplest among them is the determination of moisture and oil content (Mousseri et al., 1974, Leung et al., 1976; Miller et al., 1980; Brosio et al., 1978; Rollwitz \& Persyn, 1971). But the NMR response many times is not clear and poses problems especially when constituents other than water are present in the material (Steinberg \& Richardson, 1986). Besides the established relationship between the moisture and output of NMR experiments, various other facts helpful in determining the quality of food materials without destroying them are available in the literature: selections of chocolate confectionary products can be made non-invasively by three-dimensional magnetic resonance imaging (Miquel et al., 1998); using a spin echo pulse sequence, $128 \times 64 \times 64$ data sets were acquired with either a 5 - or $20-\mathrm{ms}$ echo time, 500-ms repetition time and four signal averages, in total 2-h scan time. Such images localize and distinguish between the constituents, and visualize both the internal and external structure of matter.

Most perishable food products are now marketed in packaged form. To increase the marketability longer shelf life is needed and this is achieved by freezing and secondary processing of the food. During freezing it is natural that ice will form within the food that may change its characteristics. Ice formation during food freezing can be examined using the NMRI method as the formation of ice has been seen to reduce the spatially located NMR signal. The characteristics of a food can be better controlled as MRI can serve to assess freezing times and the food structure during the freezing process (Kerr et al., 1998). The secondary processing changes almost all characteristics of a food, such as physical and aerodynamic (Jha \& Kachru, 1998), thermal and hygroscopic properties (Jha \& Prasad, 1993; Jha, 1999), 
which in turn, change its key acceptability factors, i.e. sensory texture and taste. The sensory texture of cooked food such as potatoes has been predicted using the NMRI technique (Thybo et al., 2000). In addition, NMR image intensity, the ratio of the oil and water resonance peaks of the one-dimensional NMR spectrum, and both the spin-lattice relaxation time and spin-spin relaxation time of water in the fruit are correlated with maturity of a fruit like avocado before harvesting (Chen et al., 1993). This important finding has desirable features for high speed sorting using a surface-coil NMR probe that determine the oil/water resonance peak ratio of the signal from one region in an intact fruit.

An on-line nuclear magnetic resonance quality evaluation sensor has recently been designed, constructed and tested (Kim et al., 1999). The device consists of a super-conducting magnet with a $20 \mathrm{~mm}$ diameter surface coil and a $150 \mathrm{~mm}$ diameter imaging coil coupled to a conveyor system. The NMR spectra of avocado fruits and one-dimensional magnetic resonance images of fresh cherries were acquired by this system. These spectra were used to measure the oil/water ratio in avocados and this ratio correlated to percent dry weight. One dimensional magnetic resonance images of cherries were later used to detect the presence of pits inside.

\section{X-ray and computerized tomography (CT)}

$\mathrm{X}$-ray imaging is an established technique to detect strongly attenuating materials and has been applied to a number of inspection applications within the agricultural and food industries. In particular, there are many applications within the biological sciences where we wish to detect weakly attenuating materials against similar background material.

X-ray computed tomography (CT) has been used to image interior regions of apples with varying moisture and, to a limited extent, density states (Tollner et al., 1992). The images were actually maps of x-ray absorption of fruit cross sections. X-ray absorption properties were evaluated using normal apples alternatively canned and sequentially freeze-dried, fruit affected by water core disorder, and normal apples freeze-dried to varying levels. The results suggested that internal differences in x-ray absorption within scans of fruit cross-sections are largely associated with differences in volumetric water content. Similarly, the physiological constituents have been monitored in peaches by CT methods in which $\mathrm{x}$-ray absorbed by the peaches is expressed in CT number and used as an index for measuring the changes in internal quality of the fruit (Barcelon et al., 1999). Relationships between the CT number and the physiological contents were determined and it was concluded that X-ray CT imaging could be an effective tool in the evaluation of peach internal quality. In another study, the potential for Compton scattered x-rays in food inspection was evaluated by imaging the density variation across a food material by measuring the Compton scatter profile across polystyrene spheres with internal voids (MacFarlane et al., 2000). In this study particular attention was paid to simulate the obscuring influence of multiple scatter. The simulated result was found to be in close agreement with the experimental observation. Some experimental test sample of a Perspex block with various embedded soft materials showed that care should be taken to ensure that the transmission image is taken with $\mathrm{X}$-rays within an appropriate energy range (Zwiggelaar et al., 1997). For low Z materials the contrasts between the materials became more pro- nounced at lower x-ray energies. If more than one soft material has to be distinguished from the surrounding area it may be advantageous to image over a range of $\mathrm{x}$-ray energies.

\section{Near-infrared spectroscopy}

The use of near-infrared spectroscopy as a rapid and often nondestructive technique for measuring the composition of biological materials has been demonstrated for many commodities. This method is no longer new; as it started in early 1970 in Japan (Kawano, 1998), just after some reports from America. Even an official method to determine the protein content of wheat is available (AACC, 1983). The National Food Research Institute (NFRI), Tsukuba has since become a leading institute in NIR research in Japan and has played a pivotal role in expanding near-infrared spectroscopy technology all over the country (Iwamoto et al., 1995). In Japan, NIR as a nondestructive method for quality evaluation was started for the determination of sugar content in intact peaches, Satsuma orange and similar other soluble solids (Kawano, 1994).

To determine the solid content of cantaloupe Dull et al. (1989) used NIR light at $884 \mathrm{~nm}$ and $913 \mathrm{~nm}$. Initially the correlation of their findings was poor mainly due to light losses. Later, Dull and Birth (1989) modified the earlier method and applied it to honeydew melons; the improved method showed better correlation. Similarly, a nondestructive optical method for determining the internal quality of intact peaches and nectarines was investigated (Slaughter, 1995). Based upon visible and near-infrared spectrophotometer techniques, the method was capable of simultaneously predicting the soluble solid content, sucrose content, sorbitol content, etc. of intact peaches and nectarines, and required no sample preparation.

Now various NIR spectrometers are available and are being used commercially. Some modifications in these available spectrometers, especially for holding the intact samples, are reported (Kawano et al., 1992; 1993). In the same sample holder a test tube for holding liquid food such as milk was also used to determine fat content (Chen et al., 1999). Recently a low cost NIR spectrometer has been used to estimate the soluble solids and dry matter content of kiwifruit (Osborne \& Kunnemeyer, 1999). Errors are within the permissible limit and the time required for obtaining data has been reduced. The influence of sample temperature on the NIR calibration equation was also evaluated and a compensation curve for the sample temperature was developed (Kawano et al., 1995) to rectify the result.

\section{Miscellaneous techniques}

Quality attributes such as invisible surface bruises, color, gloss, firmness, density, volume expansion of processed food, etc. are also important (Jha \& Prasad, 1996). Often consumers select food materials, particularly fruits and vegetables by judging these parameters visually. Multiple efforts have been made to determine these properties. A fluorescence technique was used to detect invisible surface bruises on Satsuma mandarins (Uozumi et al., 1987). The authors have also tested this method successfully to know the freshness of cucumbers and eggs and found it very useful for detecting the freshness of agricultural products.

Matsuoka et al. (1995) measured the gloss of eggplant by a spectral radiometer system and found it to be a viable parameter for determining freshness. They observed remarkable change in 
relative spectral reflectance values after $48 \mathrm{~h}$. Later, they compared their evaluation by eye in a sorting house with the integrated results of relative spectral reflectance in the visible range and found that the gloss on the surface differs with light and is caused by round and adhesives substances on the epidermal cells (Matsuoka et al., 1996). A unique gloss meter for measuring the gloss of curved surfaces was used in parallel with a conventional, flat surface gloss meter to measure peel gloss of ripening banana (Ward \& Nussinovitch, 1996). Usually banana ripeness is judged by the color of the peel. The new gloss meter is able to measure the peel correctly which helps in predicting the correct time and level of ripening. This is also able to measure the gloss of other fruits and vegetables such as green bell pepper, orange, tomato, eggplant and onion (Nussinovitch et al., 1996).

Glossiness and color, in fact, are the only visual attributes for measuring the quality of fruits and vegetables. Another property that helps a consumer in deciding the quality is firmness. Takao (1988) developed a fruit hardness tester that can measure the firmness of kiwifruit nondestructively. The tester is called a 'HIT counter' after the three words, hardness, immaturity and texture. By just setting the sample in the tester, the amount of change in shape is measured and a digital reading within a few seconds indicates about the freshness. Based on the same principle another on-line prototype HIT counter, fruit hardness sorting machine has also been developed (Takao \& Omori, 1991). The relationship between density and internal quality of watermelon can also be determined. An optimum range of density was first determined and then a new automatic density sorting system was developed to measure the hollowness of a watermelon (Kato, 1997). This system enables low density watermelons with cavities or deteriorated porous flesh to be removed and permits estimation of the soluble solid content of this fruits.

Neural networks have lately gained in popularity as an alternative to regression models to characterize the biological processes. Their decision-making capabilities can best be used in image analysis of biological products where the shape and size classification is not governed by any mathematical function. Many neural network classifiers have been used and evaluated for classifying agricultural products, but multi-layer neural network classifiers perform such tasks best (Jayas et al., 2000). Recently one scientist used a gamma-absorption technique combined with a scanning device for continuous non-destructive crop mass and growth measurement in the field (Gutezeit, 2000). Most important in this study was the accuracy of the measurement which was found to be in agreement with the direct weighing system. This method has made it possible to assess the reaction of plants and their dependence on environmental factors by growth analysis.

\section{Conclusions}

Determination of quality of any food material is actually a complex problem that requires a variety of specific sensors, more than an accumulation of simple sensors. Various techniques are being tried. NMR, X-ray CT and NIR techniques may be useful for a large volume of work in agriculture, especially for evaluation of qualities such as maturity, internal quality of fruit and conditions of food materials after processing. These techniques, although give a correct picture and precise measurement of parameters, are not convenient for small businesses. Their high cost restricts application to large entrepreneurs and developed countries only.

Two examples of the use of x-ray imaging relevant to the agricultural and food industries have been given, notably in the inspection of vegetables and food materials using low energy $\mathrm{x}$ ray imaging and in the inspection and control of dynamic processes. The X-ray imaging results have been compared with the full three dimensional information obtained by computer tomography. The CT results show more detail in the test sample than the single transmission image and remove any ambiguity. This technique would be very valuable in the inspection of materials of variable shapes usually encountered in the agricultural and food industry. The imaging techniques MRI and X-ray CT are able to show only the internal structure of the material, not the compositional or nutritional details, whereas NIR techniques are very successfully being used to determine the compositional quality of a food. However, it is not yet possible to produce an image of the internal physical quality of fruits and vegetables. All techniques are costly and their handling requires scientific knowledge. Research must continue to focus on making their use easier and lowering the cost to make it within the reach of common businesses /growers of both developed and developing countries.

Acknowledgement A special thanks is due to the Japanese Society for the Promotion of Science (JSPS) for a research fellowship grant to the authors.

\section{References}

American Association of Cereal Chemists. (1983). Approved methods of the Am. Assoc. of Cereal Chemists, 8th Ed. (March). St. Paul, Minn.

Barcelon, E.G., Tojo, S. and Watanabe, K. (1999). X-ray computed tomography for internal quality evaluation of peaches. J. Agric. Eng. Res., 73, 323-330.

Brosio, E.F., Conti, C.L. and Sykara, S. (1978). Moisture determination in starch-rich food products by pulsed nuclear magnetic resonance. J. Food Technol., 13, 107-116.

Chen, J.Y., Iyo, C. and Kawano, S. (1999). Development of calibration with sample cell compensation for determining the fat content of unhomogenised raw milk by a simple near infrared transmittance method. J. Near Infrared Spectrosc., 7, 265-273.

Chen, P., McCarthy, M.J., Kauten E., Sarig, Y. and Han, S. (1993). Maturity evaluation of avocados by NMR methods. J. Agric. Eng. Res., 55, 177-187.

Dull, G.G. and Birth, G.S. (1989). Nondestructive evaluation of fruit quality: Use of near infrared spectrophotometry to measure soluble solids in intact honeydew melons. Hortscience, 24, 754.

Dull, G.G., Birth, G.S, Smittle, D.A. and Leffler, R.G. (1989). Near infrared analysis of soluble solids in intact cantaloupe. J. Food Sci., 54, 393-395.

Farrar, T.C. and Becker, E.D. (1971). Pulse and Fourier Transform NMR: Introduction to Theory and Methods. Academic Press, New York.

Fukushima, E. and Roeder, S.B.W. (1981). Experimental Pulse NMR. Addison-Wesley Publishing Company, Reading, MA.

Gutezeit, B. (2000). Non-destructive measurement of fresh plant mass by the gamma-scanning technique applied to broccoli. J. Agric. Eng. Res., 75, 251-255.

Iwamoto, M., Kawano, S. and Yukihiro, O. (1995). An overview of research and development of near infrared spectroscopy in Japan. $J$. Near Infrared Spectrosc., 3, 179-189.

Jayas, D.S., Paliwal, J and Visen, N.S. (2000). Multi-layer neural networks for image analysis of agricultural products. J. Agric. Eng. Res. (in press).

Jha, S.N. (1999). Physical and hygroscopic properties of makhana. J. Agric. Eng. Res., 72, 145-150.

Jha, S.N. and Kachru, R.P. (1998). Physical and aerodynamic proper- 
ties of makhana. J. Food Process. Eng., 79, 301-316.

Jha, S.N. and Prasad, S. (1993). Physical and thermal properties of gorgon nut. J. Food Process Eng., 16, 237-245.

Jha, S.N. and Prasad, S. (1996). Determination of processing conditions of gorgon nut (Euryale ferox). J. Agric. Eng. Res., 63, 103112.

Kato, K. (1997). Electrical density sorting and estimation of soluble solids content of watermelon. J. Agric. Eng. Res., 67, 161-170.

Kawano, S. (1994). Non-destructive near infrared quality evaluation of fruits and vegetables in Japan. NIR News, 5, 10-12.

Kawano, S. (1998). New application of nondestructive methods for quality evaluation of fruits and vegetables in Japan. J. Jpn. Soc. Hort. Sci., 67, 1176-1179.

Kawano, S., Abe, H. and Iwamoto, M. (1995). Development of a calibration equation with temperature compensation for determining the brix value in intact peaches. J. Near Infrared Spectrosc., 3, 211218.

Kawano, S., Fujiwara, T., and Iwamoto, M.C. (1993). Nondestructive determination of sugar content in satsuma mandarin using NIR transmittance. J. Jpn. Soc. Hort. Sci., 62, 465-470.

Kawano, S., Watanabe, H. and Iwamoto, M. (1992). Determination of suger content in intact peaches by near infrared spectroscopy with fibre optics in interactance mode. J. Jpn. Soc. Hort. Sci., 61, 445451 .

Kerr, W.L., Kauten, R.J., McCarthy, M.J. and Reid, D.S. (1998). Monitoring the formation of ice during food freezing by magnetic resonance imaging. Food Sci. Technol., 31, 215-220.

Kim, S.M., Chen, P., McCarthy, M.J. and Zion, B. (1999). Fruit internal quality evaluation using on-line nuclear magnetic resonance sensors. J. Agric. Eng. Res., 74, 293-301.

Leung, H.K., Steinberg, M.P., Wei, L.S. and Nelson, A.I. (1976). Water binding of macromolecules determined by pulsed NMR. J. Food Sci., 41, 297-300.

Macfarlane, N.J.B., Bull, C.R., Tillett, R.D., Speller, R.D., Royle, G.J. and Johnson, K.R.A. (2000). The potential for Compton scattered xrays in food inspection: The effect of multiple scatter and sample inhomogeneity. J. Agric. Eng. Res., 75, 265-274.

Matsuoka, T., Miyauchi, K. and Sun, D. (1995). Basic studies on the quality evaluation of agricultural products-Quantification of gloss of eggplants by spectral characteristics. J. Jpn. Sci. Agric Mach., 57, $33-40$.

Matsuoka, T., Miyauchi, K. and Yano, T. (1996). Basic studies on the quality evaluation of agricultural products (Part 2)-The evaluation of colour and gloss decrease on the surface of eggplants. J. Jpn. Sci. Agric. Mach., 58, 69-77.

Miller, B.S., Lee, M.S., Hughes, J.W. and Pomernz, Y. (1980). Mea- suring high moisture content of cereal grains by pulsed nuclear magnetic resonance. Cereal Chem., 57, 126-129.

Miquel, M.E., Evans, S.D. and Hall, L.D. (1998). Three dimensional imaging of chocolate confectionary by magnetic resonance methods. Food Sci. Technol., 31, 339-343.

Mousseri, J., Steinberg, M.P., Nelson, A.I. and Wei, L.S. (1974). Bound water capacity of corn starch and its derivatives by NMR. $J$. Food Sci., 39, 114-116.

Nussinovitch, A., Ward, G. and Mey-Tal, E. (1996). Gloss of fruits and vegetables. Food Sci. Technol., 29, 184-186.

Osborne, S.D. and Kunnemeyer, R. (1999). A low cost system for the grading of kiwifruit. J. Near Infrared Spectrosc., 7, 9-15.

Rollwitz, W.L. (1984). Radio frequency spectroscopy: Versatile sensors for agriculture applications. ASAE Paper No. 84-1590, ASAE, St. Joseph, MI 49085.

Rollwitz, W.L. and Persyn, G.A. (1971). On-stream NMR measurements and control. Am. Oil Chem. Soc. J., 48, 59-66.

Slaughter, D.C. (1995). Nondestructive determination of internal quality in peaches and nectarines. Trans. ASAE, 38, 617-623.

Steinberg, M.P. and Richardson, S.J. (1986). Application of nuclear magnetic resonance. Paper No. 11. Presented at the International Union Food Science and Technology Symposium on Water Activity: Theory and Application. June 13-14, Dallas, TX.

Takao, H. (1988). HIT counter. Noryu Giken Kaihou, 180, 7-9 (in Japanese).

Takao, H. and Omori, S. (1991). Quality evaluation of fruits and vegetables using light transmittance. Noryu Giken Kaihou, 145, 14-16 (in Japanese).

Thybo, A.K., Bechmann, I.E., Martens, M. and Engelsen, S.B. (2000). Prediction of sensory texture of cooked potatoes using uniaxial compression, near infrared spectroscopy and low field $\mathrm{H}$ NMR Spectroscopy. Food Sci. Technol., 33, 103-111.

Tollner, E.W., Hung, Y.-C., Upchurch, B.L. and Prussia, S.E. (1992). Relating $\mathrm{X}$-ray absorption to density and water content in apples. Trans. ASAE, 35, 1921-1928.

Uozumi, J., Kawano, S., Iwamoto, M. and Nishinari, K. (1987). Spectrophotometric system for the quality evaluation of unevenly couloured food. Nippon Shokuhin Kogyo Gakkaishi, 34, 163-170 (in Japanese).

Ward, G. and Nussinovitch, A. (1996). Peel as a potential indicator of banana ripeness. Food Sci. Technol., 29, 289-294.

Zwiggelaar, R., Bull, C.R., Mooney, M.J. and Czarnes, S. (1997). The detection of soft materials by selective energy $\mathrm{x}$-ray transmission imaging and computer tomography. J. Agric. Eng. Res., 66, 203212. 